\title{
HANDBUCH DER \\ VERMESSUNGSKUNDE
}

von

\author{
DR. PHIL. h. c. W. JORDAN
}

weiland Professor an der Technischen Hochschule Hannover

\begin{abstract}
DRITTER BAND
Zweiter Halbband

SPHAROIDISCHE BERECHNUNGEN, KONFORME ABBILDUNG DES ERDELLIPSOIDS

UND AUFGABEN DER ERDMESSUNG
\end{abstract}

Mit zahlreichen Abbildungen

\author{
Bearbeitet von \\ DR. DR.-ING. e.h. O. EGGERT \\ weiland Professor an der Technischen Hochschule Berlin \\ Direktor des Preußischen Geodätischen Instituts in Potsdam \\ Neunte Auflage \\ (Unveränderter Abdruck der erweiterten achten Auflage)
}

STUTTGART

J. B. METZLER SCHE VERLAGSBUCHHANDLUNG 
Dr. Dr.-Ing.e.h: Otto Eggert,

geboren am 4. Februar 1874 in Tilsit,

gestorben am 20. Januar 1944 in Danzig

ISBN 978-3-476-99868-2

ISBN 978-3-476-99867-5 (eBook)

DOI 10.1007/978-3-476-99867-5

(C) 1941 Springer-Verlag GmbH Deutschland

Ursprünglich erschienen bei J.B. Metziersche Verlagsbuchhandlung, Stuttgart in 1941 


\section{Vorwort.}

Mit dem vorliegenden zweiten Halbband des dritten Bandes ist eine neue Auflage des gesamten Handbuchs der Vermessungskunde zum AbschluB gebracht. Der Halbband enthält im ersten Teil die geodätischen Berechnungen auf dem Erdellipsoid sowie die geodätisch wichtigen Abbildungen des Erdellipsoids und im zweiten Teil die Aufgaben der Erdmessung.

Die Berechnungen auf dem Erdellipsoid sind im wesentlichen in der bisherigen Form dargestellt, jedoch ist die Theorie der geodätischen Linie mathematisch etwas schärfer gefaßt worden. Für die Übertragung geographischer Koordinaten sind die jetzt weitverbreiteten Formeln von Schreiber abgeleitet, ferner sind die sphäroidischen Mittelbreitenformeln zum Teil in eine andere Forrn gebracht worden.

Außer den Soldnerschen rechtwinkligen Koordinaten ist entsprechend dem Inhalt des ersten Halbbandes die Theorie der querachsigen rechtwinkligen Koordinaten entwickelt.

Wesentlich umgestaltet sind die Kapitel, die die Abbildung des Erdellipsoids behandeln. Unter Einführung der isometrischen Breite werden die allgemeinen mathematischen Grundlagen für die Theorie der konformen $\mathrm{Ab}$ bildung des Erdellipsoids entwickelt. Hieran schließt sich die ausführliche Behandlung der Gauß-Krügerschen Projektion, wobei die Koordinatenumformung für zwei Gauß-Krügersche Systeme neu hinzugekommen ist.

Als weitere Anwendung der konformen Abbildung folgt die konforme querachsige Projektion, die konforme Kegelprojektion und in kurzer Darstellung die stereographische Projektion von Roussilhe.

Bei der vielfachen Anwendung der konformen Doppelprojektion in verschiedenen Ländern wurde die Theorie der konformen Abbildung des Ellipsoids auf die Kugel im bisherigen Umfang beibehalten und zum Teil mathematisch vertieft. Dagegen wurde die frïhere preußische konforme Doppelprojektion, nachdem sie zugunsten der für das ganze Reichsgebiet eingeführten Gauß-Kriigerschen Projektion aufgegeben worden ist, nur noch kurz behandelt.

Im zweiten Teil des Halbbands ist vor allem den vielen Neuerungen in der Methode der Schweremessung Rechnung getragen worden. So sind neue Pendelapparate für relative Schweremessungen beschrieben, ferner ist auf die 
Pendelmessungen im Unterseeboot und auf die Theorie des Pendels von Holweck-Lejay eingegangen. Ein besonderer Abschnitt ist den statischen Schweremessern gewidmet, die zur Zeit eine ständig wachsende Bedeutung gewinnen. Eine weitere Ergänzung hat auch die Reduktion der Schweremessungen, insbesondere unter Berücksichtigung der Isostasie, gefunden.

Für die Ausgleichung astronomisch-geodätischer Netze ist gezeigt, wic solche Ausgleichungen in strenger Form ausgeführt werden können. Außerdem wurde die Bedeutung der Laplaceschen Gleichung für eine Landestriangulation mehr hervorgehoben.

Im letzten Kapitel wurden, um die Grenzen der eigentlichen Geodäsie nicht wesentlich zu überschreiten, lediglich die neueren Ergebnisse der Polbewegung aufgenommen.

Für das Studium des Vermessungsingenieurs kann von den ersten Begriffen in bezug auf das Erdellipsoid von S. 1 und 2 sofort zu $\S 6$ und 7 übergegangen werden, in denen die Grundgleichungen der geodätischen Linie entwickelt sind. Hierauf können § 17-21 des Kapitels III folgen, die die wichtigsten Formelsysteme zur Utbertragung der geographischen Koordinaten enthalten. Von den rechtwinkligen Koordinaten auf dem Ellipsoid sind als wichtigste die Soldnerschen Koordinaten in $\S 27$ und 28 behandelt worden.

Übergehend zu der Abbildung des Erdellipsoids ist das Studium von $\$ 30-37$ erforderlich, in denen alles enthalten ist, was für das Verständnis der GaußKrügerschen Koordinaten erforderlich ist. Die Koordinatenumformung zwischen zwei Gauß-Krügerschen Systemen in $\S 38$ kann für späteres Studium zurückgestellt werden.

Schließlich sind noch $\S 54$ und 55 von Interesse.

Für die Fälle, in denen die Kenntnis der konformen Doppelprojektion notwendig ist, findet sich alles Erforderliche in $\S 46-50$ und 52-53.

Nach Abschluß dieses ersten Lehrgangs der Landesvermessung wäre § 88-96 durchzuarbeiten, um die Lehre von den Lotabweichungen für die Landesvermessung nutzbar machen zu können.

Alles Weitere der Kapitel VI-X bezieht sich auf die Aufgaben der Erdmessung und kann dem Vermessungsingenieur zur Vertiefung seines Wissens dienen.

Berlin-Dahlem, im September 1941. 


\section{Inhaltsübersicht.}

\section{Kapitel I. \\ Normalschnitte und geodätische Linie.}

$\S$ 1. Gegennormalschnitte . . . . . . . . . . . . . . . . . . . . 1

$\S$ 2. Ellipsenbogen eines Normalschnittes . . . . . . . . . . . . . . . . . 8

§ 3. Konvergenz der beiden Normalschnitte . . . . . . . . . . . . . . . . 13

$\S$ 4. EinfluB der Höhe der Zielpunkte auf die Messung der Horizontalwinkel . 16

$\S 5$. Ubertragung von Längen, Breiten und Azimuten auf dem Ellipsoid mit Hilfe von Vertikalschnitten . . . . . . . . . . . . . . . . . . 19

§ 6. Die geodätische Linie . . . . . . . . . . . . . . . . . . . . . . . 23

$\S$ 7. Differentialgleichungen der geodätischen Linie . . . . . . . . . . . . 26

$\S$ 8. Die geodätische Linie als kürzeste Linie . . . . . . . . . . . . . . . 29

$\S$ 9. Vergleichung der geodätischen Linie mit den Normalschnitten . . . . . 33

\section{Kapitel II. \\ Sphäroidische Dreiedksberechnung.}

$\S 10$. Die reduzierte Länge der geodätischen Linie . . . . . . . . . . . . 39

$\S$ 11. Sphäroidische Polarkoordinaten . . . . . . . . . . . . . . . 44

$\S 12$. Verbindung eines rechtwinkligen Systems und eines Polarsystems . . . . 47

$\S$ 13. Reihenentwicklung für das rechtwinklige sphäroidische Drejeck . . . . . 49

$\S$ 14. Berechnung des allgemeinen (schiefwinkligen) sphäroidischen Dreiecks . . 54

$\S 15$. Krumme Oberfläche des sphäroidischen Dreiecks . . . . . . . . . . . 57

$\S 16$. Praktische Anwendung der allgemeinen Theorie der sphäroidischen Dreiecke 61

\section{Kapitel III. \\ Sphäroidische Koordinaten.}

§ 17. Sphäroidisches Polardreieck . . . . . . . . . . . . . . . . . . . . . 67

$\S 18$. Reihenentwicklungen nach Potenzen von $s \ldots \ldots$. . . . . . . 69

$\S$ 19. Die Formeln von Schreiber zur Ubertragung geographischer Koordinaten 76

§ 20. Krügers Formeln zur Übertragung geographischer Koordinaten . . . . . 82

$\S 21$. Sphäroidische Mittelbreitenformeln . . . . . . . . . . . . . 86

$\S 22$. Die reduzierte Breite . . . . . . . . . . . . . . . . . . . . 97

$\S 23$. Das sphärische Hilfsdreieck mit reduzierten Breiten . . . . . . . . . . 102

$\S 24$. Integration der Differentialgleichungen des Polardreiecks . . . . . . . . 104

$\S 25$. Bessels Formeln zur Ubertragung geographischer Koordinaten . . . . . 107

$\S 26$. Jordans Auflösung des geodätischen Polardreiecks . . . . . . . . . . . 111

$\S 27$. Rechtwinklige Koordinaten auf dem Ellipsoid . . . . . . . . . . . 117

$\S 28$. Potenzreihen für rechtwinklige und geographische Koordinaten . . . . . 128

§ 29. Rechtwinklige querachsige Koordinaten . . . . . . . . . . . 135

Kapitel IV.

Die Abbildung des Erdellipsoids.

§30. Die Grundgleichungen der Abbildung . . . . . . . . . . . . . . 142

$\S 31$. Die konforme Abbildung des Erdellipsoids . . . . . . . . . . . . . . 146

§ 32. Die Gauß-Krügersche Projektion . . . . . . . . . . . . . . . . . . . 150 
Inhaltsübersicht.

$\S 33$. Berechnung der geographischen Koordinaten aus den konformen ebenen Koordinaten . . . . . . . . . . . . . . . . 154

$\S 34$. Meridiankonvergenz und Vergrößerungsverhältnis. Potenzreihen . . . . . 159

$\S$ 35. Zusammenstellung der Formeln und Zahlenbeispiele . . . . . . . . . . 166

§36. Krügers Formeln für geographische Koordinaten und konforme ebene Koordinaten . . . . . . . . . . . . . 168

§37. Entfernungs- und Richtungsreduktion . . . . . . . . . . . . . . 175

$\S 38$. Koordinatenumformung für zwei Gauß-Krügersche Systeme . . . . . . 184

§39. Querachsige konforme Koordinaten . . . . . . . . . . . . . . 195

$\S 40$. Die Meridiankonvergenz und das Vergrōßenungsverhältnis der querachsigen Projektion . . . . . . . . . . . . . . . . . . 201

§ 41. Konforme Kegelprojektion .................... 204

$\S$ 42. Reihenentwicklung für die konforme Kegelprojektion . . . . . . . . . 207

$\S$ 43. Reihenentwicklungen für die rechtwinkligen Koordinaten . . . . . . . 210

§44. Konforme Kegelprojektion mit zwei Grundparallelkreisen . . . . . . . 216

$\S$ 45. Die stereographische Projektion . . . . . . . . . . . . . . . . . . 218

Kapitel V.

Konforme Abbildung des Ellipsoids auf die Kugel und konforme Doppelprojektion.

§ 46. Grundformeln . . . . . . . . . . . . . . . . . . . . . . . . . 222

§ 47. Wahi der Konstanten .................. 225

$\S$ 48. Reihenentwicklung für die Breitendifferenz . . . . . . . . . . . . . . 229

$\S 49$. Reihenentwicklung für das Vergrößerungsverhältnis . . . . . . . . . . 233

$\S 50$. Azimutreduktion . . . . . . . . . . . . . . . . . . . 236

$\S 51$. Allgemeine Beziehurg zwischen dem Vergrößerungsverhältnis $m$ und dem Krümmungsdifferential der Abbildung . . . . . . . . . . . . . . . . 241

§52. Hilfstafeln und Zahlenbeispiele . . . . . . . . . . . . . . . . . . . 243

§53. Konforme Doppelprojektion ................... 245

§54. Karten mit geographischen Netzlinien ... . . . . . . . . . . 250

$\S 55$. Die rechtwinkligen Koordinatensysteme des Deutschen Reichs . . . . . 260

Kapitel VI.

Bestimmung der Dimensionen des Erdellipsoids durch Gradmessungen.

$\S 56$. Bestimmung der Meridianellipse durch zwei Breitengradmessungen . . . 272

$\S 57$. Reduktion eines Gradmessungsbogens auf den Meridian . . . . . . . . 274

§58. Ausgleichung mehrerer Breitengradmessungen . . . . . . . . . . . . . 279

\$59. Iängengradmessung . . . . . . . . . . . . . . . . . . . . . . . . 283

$\S 60$. Azimutübertragung . . . . . . . . . . . . . . . . . . . . . 284

\section{Kapitel VII.}

Die mathematische Erdgestalt und die Sdwwerkraft.

$\S 61$. Anziehung eines Massenpunkts durch den Erdkörper . . . . . . . . . 288

§62. Gleichgewicht eines starren Punktsystems . . . . . . . . . . . . . . 292

$\S 63$. Reihenentwicklung für das Potential der Schwerkraft . . . . . . . . . 294

$\S 64$. Das Theorem von Clairaut . . . . . . . . . . . . . . . . . . . 297 
\$65. Die Gestalt der Sphäroidflächen . . . . . . . . . . . . . . . . . 303

$\S 66$. Bestimmung der großen Halbachse des Erdellipsoids aus der Mondparallaxe und der Schwerkraft . . . . . . . . . . . . . . 306

\$ 67. Bestimmung der Abplattung der Erde aus den UnregelmäBigkeiten der Mondbewegung .

\section{Kapitel VIII. \\ Die Messung der Schwerkraft.}

§68. Das mathematische Pendel . . . . . . . . . . . . . . . . 311

$\S 69$. Das physische Pendel . . . . . . . . . . . . . . . . . . . 315

$\S 70$. Schwingung eines physischen Pendels um eine zylindrische Achse . . . . 321

$\S 71$. Das Mitschwingen des Pendelstativs . . . . . . . . . . . . 324

§ 72. Ältere Pendelmessungen . . . . . . . . . . . . . . . . . . . . 329

§ 73. Der Repsoldsche Pendelapparat . . . . . . . . . . . . . . 338

$\S 74$. Bestimmung des Mitschwingens des Stativs............. 344

$\S 75$. Zahlenbeispiel . . . . . . . . . . . . . . . . . . . . . 349

$\S 76$. Der Sternecksche Pendelapparat . . . . . . . . . . . . . . 357

$\S$ 77. Das Mitschwingen des Sterneckschen Pendelstativs . . . . . . . . . 363

$\$$ 78. Reduktion der Pendelmessungen. EinfluB der Luftdichte und der Pendeltemperatur .................. . . 368

$\S$ 79. Besondere Methoden der Pendelmessung ... . . . . . . . . 372

\$8. Die statischen Schweremesser . . . . . . . . . . . . . . 377

81. Reduktion der Schweremessungen ............. . 383

$\S 82$. Die isostatische Reduktion der Schweremessungen . . . . . . . . . 387

\$83. Die normale Schwerkraft im Meeresniveau . . . . . . . . . . . . . 392

\$ 84. Messungen vermittelst der Drehwaage ... . . . . . . . . . . . 395

$\S 85$. Die Verwertung der Drehwaagenmessungen . . . . . . . . . . . . 403

$\S 86$. Theorie des geometrischen Nivellements . . . . . . . . . . . 408

$\S 87$. Die orthometrische Verbesserung des Nivellements . . . . . . . . 412

\section{Kapitel IX. \\ Lotabweichungen.}

$\S$ 88. Allgemeines über Lotabweichungen . . . . . . . . . . .

$\S$ 89. Grundformeln für die Berechnung der Lotabweichungen aus astronomischen und geodätischen Messungen ................ 417

$\S 90$. Differentialformeln für die geodätische Linie . . . . . . . . . . . 422

$\S$ 91. Einfluß einer Änderung des Ellipsoidkonstanten . . . . . . . . . . . 430

$\S 92$. Ausgleichung eines astronomisch-geodätischen Netzes . . . . . . . . . 435

$\S$ 93. Beispiel einer Netzausgleichung . . . . . . . . . . . . . . . 439

$\S 94$. Bestimmung der Gewichte und Ausgleichung . . . . . . . . . . . . 446

$\S 95$. Strenge Ausgleichung des astronomisch-geodätischen Netzes . . . . . 453

$\S$ 96. Bestimmung der Orientierung eines Dreiecksnetzes . . . . . . . . 457

$\S$ 97. Berechnung der Dimensionen des Erdsphäroids . . . . . . . . . . 459

$\S$ 98. Bestimmung von Geoidprofilen und kleiner Teile der Geoidfläche . . . 468

$\S 99$. Messung von Lotabweichungen vermittelst der Drehwaage . . . . . 475

$\S 100$. Die trigonometrische Höhenmessung . . . . . . . . . . 482 


\section{Kapitel X.}

\section{Periodische Lotstörungen und die Polbewegung.}

$\S 101$. Veränderlichkeit der Lotrichtung unter dem Einfluß von Sonne und Mond 487 $\S$ 102. Das Horizontalpendel . . . . . . . . . . . . . . . . . . . . . 492 § 103. Reihenentwicklung für das Potential der fluterzeugenden Kraft . . . . 498 § 104. Berechnung der Lotbewegungen ... . . . . . . . . . . . . . . . 504 $\S 105$. Die Auswertung der Horizontalpendelmessungen . . . . . . . . . 506 $\S$ 106. Bestimmung der Elastizität der Erde . . . . . . . . . . . . . . . 512 $\S$ 107. Die Rotation der Erde . . . . . . . . . . . . . . . . . 516 \$ 108. Der Internationale Breitendienst . . . . . . . . . . . . . . . . 520 $\S$ 109. Bestimmung der Elastizität der Erde aus der Polbewegung . . . . . 527

\section{Drudkfehler in Band III, 1. Halbband 1939.}

Seite 143 Zeile 8 von unten soll (15) statt (10).

Seite 217 Zeile 18 soll $3 b c^{2}$ statt $6 b c^{2}$.

Seite 248 Zeile 16 soll Mittlerer Krümmungshalbmesser $r$ statt Krümmungsverhältnis $N: M$.

Seite 260 Formel (5) soll $\frac{1}{2} \sin 2 \varphi$ statt $\frac{1}{2} \cos 2 \varphi$.

Seite 284 Zejle 8 soll $C A^{\prime} B^{\prime}$ statt $C A^{\prime} B$.

Seite 329 Zeile 11 soll $\left.\frac{y^{3}}{3 r^{3}}\right) \operatorname{tang} \varphi_{2}$ statt $\left.\frac{y^{3}}{3 r^{3}} \operatorname{tang} \varphi_{2}\right)$.

Seite 335 In der Mitte der mittleren Spalte soll $\sin ^{2} \varphi$ statt $\sin \varphi$.

Seite 336 Dritter Abschnitt, letzte Spalte soll $\left(b^{\prime}\right) \mid 4.3668_{n}$ statt $(b) \mid 4.3668_{n}$.

Vierter Abschnitt, rechts unten soll $\left(d^{\prime}\right)=-0,0043$ statt $(d)=-0,0043$.

Seite 379 Zeile 3 von unten soll $\varphi_{0}=0$ statt $\varphi=0$.

Seite 390 Zeile 20 von unten soll Ebene statt Kugelfläche.

Seite 396 Zeile 10 soll $+\frac{B s^{2}}{6}$ statt $\frac{B s^{2}}{2}$.

Seite 397 Zeile 1 soll $=\frac{A s}{2} \xi+\frac{B s^{2}}{6} \xi$ statt $\frac{A s}{6} \xi+\frac{B s^{2}}{6} \xi$.

Seite 399 Zeile 2 soll $\frac{\mathfrak{y}^{3}}{6 r^{2}}$ statt $\frac{\mathfrak{h}^{2}}{6 r^{2}}$.

Seite 399 Zeile 20 soll $+15278,872$ statt $-15278,872$.

Seite 429 Zeile 14 soll $\lambda \sin \varphi_{0}+\frac{1}{3}\left(\frac{y^{3}}{R_{0}{ }^{3}}+\frac{3 y^{5} x}{R_{0}{ }^{4}}\right)=\operatorname{statt} \lambda \sin \varphi_{0}=\frac{1}{3}\left(\frac{y^{3}}{R_{0}{ }^{3}}+\frac{3 y^{3} x}{R_{0}{ }^{4}}\right)=$

Seite 431 Zeile 9 von unten soll $\frac{\mu x^{4}}{24 r^{4}}$ statt $\frac{\mu}{24 r^{6}}$.

Seite 435 Zeile 14, letztes Glied soll $-\frac{t_{0}}{4 r^{3}} y_{1}{ }^{2}\left(y_{2}-y_{1}\right)$ statt $-\frac{t_{0}}{4 r^{3}} y^{2}\left(y_{2}-y_{1}\right)$

Seite 435 Zeile 10 von unten soll $-\frac{t_{0} \varrho}{12 r^{3}}$ statt $+\frac{t_{0} \varrho}{12 r^{3}}$.

Seite 435 Zeile 9 von unten soll $-\frac{t_{0} \varrho}{24 r^{3}}$ statt $+\frac{t_{0} \varrho}{24 r^{3}}$.

Seite 477 Zeile 18 von unten soll $\alpha=14^{h} 12^{m}$ statt $14^{h} 42^{m}$.

Seite 477 Zeile 13 von unten soll $a^{\prime}=14^{h} 12^{m}$ statt $14^{h} 42^{m}$.

An hang.

Seite [56] Zeile 4 soll $|.02| .03 \mid$ usw. $|.09|$ statt $|0.2| 0.3 \mid$ usw. $|0.9|$.

Seite [56] Zeile 15 soll 269.1 statt 169.1 . 